
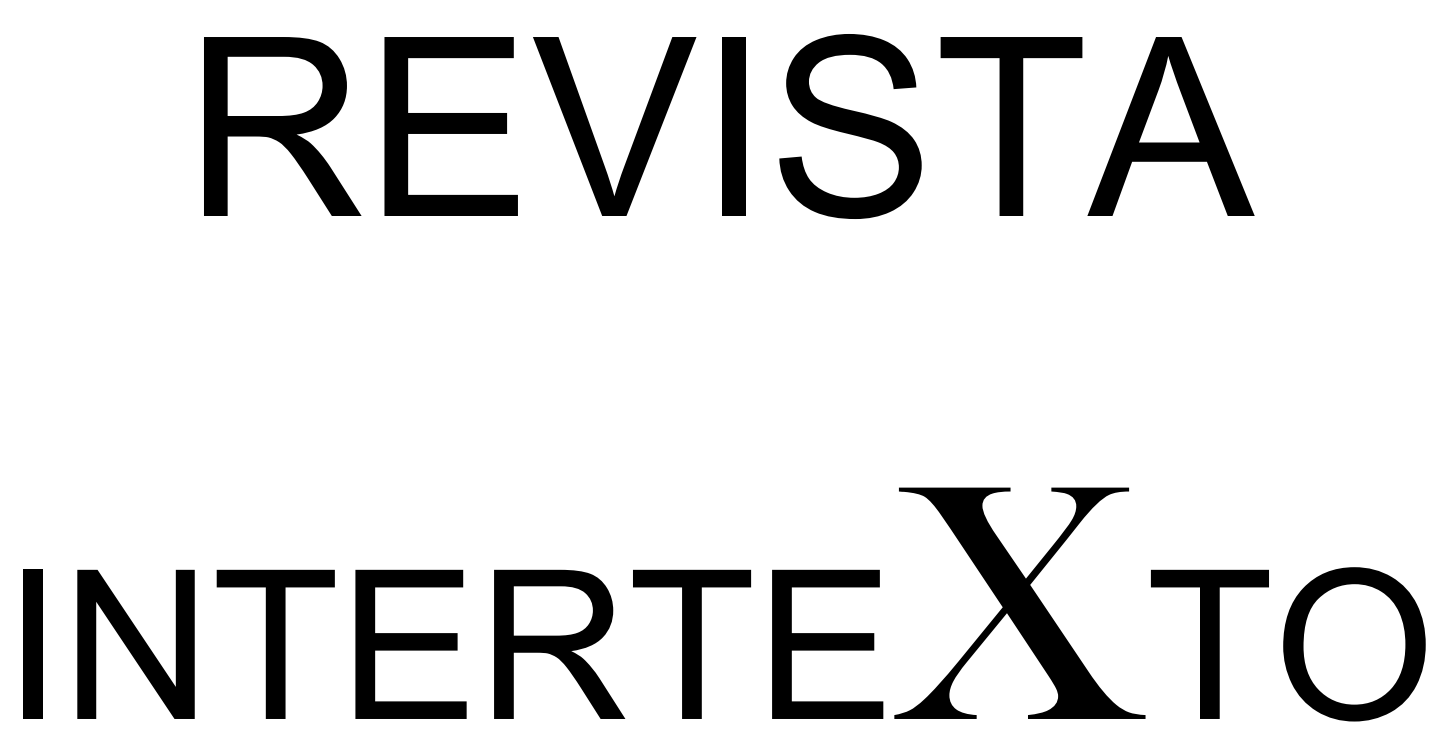


\begin{tabular}{|l|l|l|l|l|l|l|}
\hline InterteXto & Uberaba & UFTM & $\begin{array}{l}\text { v. 4 } \\
\text { n. } 1\end{array}$ & p. 01-65 & 2011 - jan. / jul. & ISSN 1981-0601 \\
\hline
\end{tabular}

\author{
Revista InterteXto \\ Curso de Letras \\ UFTM \\ Universidade Federal do Triângulo Mineiro \\ Avenida Frei Paulino, 30 - Bairro Abadia \\ CEP 38025-180 - Uberaba - MG
}

\title{
Editor responsável
}

Ozíris Borges Filho

Comissão Editorial

Acir Mario Karwoski

Carlos Francisco de Morais

Deolinda de Jesus Freire

Elizandra Zeulli

Fani Miranda Tabak

Irma Beatriz Araújo Kappel

Janaína Aguiar Mendes Galvão

Jauranice Rodrigues Cavalcanti

Juliana Bertucci Barbosa

Luciana Moura Colucci Camargo

Marinalva Vieira Barbosa

Maíra Sueco Maegava Córdula

Renata Nascimento Salgado

Sandra Eleutério Campos Martins

\section{Conselho Editorial (Pareceristas “ad hoc”)}

Adail Ubirajara Sobral (UNIMEP), Adair Bonini (UNISUL), Andréia de Fátima Rutiquewiski Gomes (UNICENTRO), Ângela Bustos Kleiman (UNICAMP), Anna Christina Bentes (UNICAMP), Antônio Fernandes (UFG), Charles Bazerman (UCSB-USA), Claudia Mendes Campos (UFPR), Clarice Zamonaro Cortez (UEM), Dermeval da Hora Oliveira (UFPB), Durvali Emilio Fregonezi (FACCAR), Eduardo Tadeu Roque Amaral (UFMG), Elvira Lopes Nascimento (UEL), Emerson da Cruz Inácio (USP), Ezequiel Theodoro da Silva (UNICAMP), Fábio José Rauen (UNISUL), lara Bemquerer Costa (UFPR), Isabel Mercadé Navarro (UPF-BARCELONA), Ivan Marcos Ribeiro (UFU), João Wanderley Geraldi (UNICAMP), Joel Cardoso (UFPA), Jorge Vicente Valentim (UFSCAR), José Luiz Fiorin (USP), José Luiz Foureaux de Sousa Júnior (UFOP), Kanavillil Rajagopalan (UNICAMP), Karim Siebeneicher Brito (FAFIUV), Latuf Isaias Mucci (UFF), Lúcia Maria de Assunção Barbosa 


\begin{tabular}{|l|l|l|l|l|l|l|}
\hline InterteXto & Uberaba & UFTM & $\begin{array}{l}\text { v. 4 } \\
\text { n. } 1\end{array}$ & p. 01-65 & 2011 - jan. / jul. & ISSN 1981-0601 \\
\hline
\end{tabular}

(UFSCAR), Luiz Carlos Travaglia (UFU), Luzia de Maria Rodrigues Reis (UFF), Márcia Elizabeth Bortone (UnB), Marcos Baltar (UCS), Maria Aparecida Garcia Lopes-Rossi (UNITAU), Maria Auxiliadora Bezerra (UFCG), Maria da Graça Costa Val (UFMG), Maria das Graças Soares Rodrigues (UFRN), Marisa Martins Gama Kalil (UFU), Orlando Vian Júnior (UNIFESP), Renilson José Menegassi (UEM), Roxane Helena Rodrigues Rojo (UNICAMP), Sidney Barbosa (UNESP-Arar), Shirley Geok-Lin Lim (UCSB - USA), Valdir Heitor Barzotto (USP), Vera Lúcia Lopes Cristóvão (UEL), Vera Lúcia Menezes de Oliveira e Paiva (UFMG).

Publicação semestral

Revista InterteXto - Organizada pelo Curso de Letras da UFTM - Universidade Federal do Triângulo Mineiro

Vol. 4 No 1 (2011) - Uberaba - MG

Publicação em meio eletrônico http://revistaintertexto.letras.uftm.edu.br/ ISSN 1981-0601

1. Literatura 2. Teoria da Literatura 3. Lingüística. 4. Lingüística Aplicada 


\begin{tabular}{|l|l|l|l|l|l|l|}
\hline InterteXto & Uberaba & UFTM & $\begin{array}{l}\text { v. 4 } \\
\text { n. } 1\end{array}$ & p. 01-65 & 2011 - jan. / jul. & ISSN 1981-0601 \\
\hline
\end{tabular}

\section{SUMÁRIO}

Estudo sobre alguns conceitos considerados fundamentais para a compreensão da teoria sócio-histórica do filósofo russo Mikhail Bakhtin: uma investigação teórica

Deize Fernandes Diniz.

O aspeamento em respostas de questão interpretativa-argumentativa Ana Maria da Silva.

Concepções de língua e linguagem em Chomsky, Benveniste e Labov Welisson Marques.

Revista de Uberaba: um esboço histórico

Taís Iniz de Paiva 